\title{
Erratum to: Biomass and nutrient accumulation by cover crops and upland rice grown in succession under no-tillage system as affected by nitrogen fertilizer rate
}

Edson Cabral da Silva ${ }^{1}$ - Takashi Muraoka ${ }^{2}$ Alefe Viana Souza Bastos ${ }^{1}$ - Vinícius Ide Franzini ${ }^{3} \cdot$ Salatiér Buzetti $^{4}$. Frederico Antonio Loureiro Soares ${ }^{1} \cdot$ Marconi Batista Teixeira $^{1} \cdot$ José Alberto Bendassolli ${ }^{2}$

Published online: 16 April 2020

(c) Korean Society of Crop Science (KSCS) and Springer Nature B.V. 2020

\section{Erratum to:}

J. Crop Sci. Biotech. 2020 (March) 23 (2):117-126

https://doi.org/10.1007/s12892-019-0288-0

Due to an unfortunate oversight the author's name and affiliation have been given erroneously. It should be read:

Vinícius Ide Franzini

Embrapa Cocais, São Luiz MA, Brazil

The authors apologies this mistake.

The original article can be found online at https://doi.org/10.1007/ s12892-019-0288-0.

Alefe Viana Souza Bastos

alefe_viana@hotmail.com

1 Department of Irrigation and Hydraulics, Instituto Federal Goiano, Rio Verde, GO, Brazil

2 Center of Nuclear Energy in Agriculture, Universidade de São Paulo, Piracicaba, SP, Brazil

3 Embrapa Cocais, São Luiz, MA, Brazil

4 Universidade Estadual Paulista "Júlio de Mesquita Filho" (FEIS/UNESP), Ilha Solteira, SP, Brazil 